\title{
Standardized reporting templates with mandatory reporting fields and "pick-list" options improve use of Prostate Imaging and Data Reporting System version 2 in clinical practice: A plan-do-study-act analysis
}

\author{
Kevin Moran ${ }^{1}$; Rodney H. Breau²; Ilias Cagiannos²; Luke T. Lavallée ${ }^{2}$; Christopher \\ Morash $^{2}$; Joseph O'Sullivan ${ }^{1}$; Nicola Schieda ${ }^{1}$ \\ ${ }^{1}$ Department of Radiology, The Ottawa Hospital, University of Ottawa, Ottawa, ON, Canada; ${ }^{2}$ Department \\ of Surgery, Division of Urology, The Ottawa Hospital, The University of Ottawa, Ottawa, ON, Canada
}

Cite as: Can Urol Assoc J 2018 November 5; Epub ahead of print. http://dx.doi.org/10.5489/cuaj.5630

Published online November 5, 2018

\section{$* * *$}

\section{Introduction}

The Prostate Imaging and Data Reporting System version 2 (PI-RADS v2) aims to simplify performance, interpretation, and reporting of prostate magnetic resonance imaging (MRI) ${ }^{1}$. PI-RADS v2 introduced probability scores (assessment categories, Table 1), which indicate the likelihood of clinically significant cancer (Gleason score $\geq 7)^{2}$ based upon MRI findings. PI-RADS v2 has been validated as accurate for detection of cancers and improves interobserver agreement ${ }^{3}$. Despite this, in our experience, use of PI-RADS v2 scores in practice is variable. This study evaluated a method to improve utilization of PI-RADS v2 scores by using a plan-do-study-act (PDSA) analysis.

\section{Methods}

This retrospective single-institution study was conducted under a waiver from the institutional review board. Our PDSA cycle included: 1) a "plan" to improve utilization of PI-RADS-version.2 scores, 2) “doing” through education (Lectures on PI-RADSversion.2 given by the Director of Prostate Imaging [BLINDED] and distributed literature highlighting PI-RADS-version.2) and providing a standardized reporting template with “pick-list” fields for PI-RADS-version.2 scores, 3) “studying” utilization and, 4) “acting” through feedback on utilization and Urologist satisfaction with reporting.

Time periods

1. Pre-intervention (January 1 to June 30, 2016): Prior to education and standardized reporting and one-year following publication of PI-RADS-version.2.

2. Intervention (July 1 to December 30, 2016): Following educational activities and creation of reporting templates. The standardized template (shown in 
Supplementary Fig. 1), included “pick-lists” (Powerscribe 360, Nuance Communications) for PI-RADS-version.2 scores under the "IMPRESSION" heading.

3. Post-intervention (January 1 to April 1, 2017): Following feedback indicating non-universal use of templates, PI-RADS-version.2 scores and Urologist dissatisfaction with non-standardized reporting.

All MRI studies were reported by fellowship-trained Radiologists ( $n=11)$. A search of our imaging archive (Horizon Medical Imaging v11, McKesson Corporation) from January 2016-April 2017 identified 619 men who underwent multi-parametric MRI at 3Tesla. After exclusion, Figure 1, 309 men were included. A Radiology Resident (BLINDED) recorded: use of reporting templates and ACs, MRI result (positive[+] or negative[-]), if targeted biopsies (TB) were performed and interval between +MRI and TB.

Statistical analysis

Comparisons were performed using the Chi-square test and ANOVA. Statistical analysis was performed using STATA v13 (Statcorp).

\section{Results}

Distribution of patients was: 35.6\% (110/309) pre-intervention, 37.2\% (115/309)

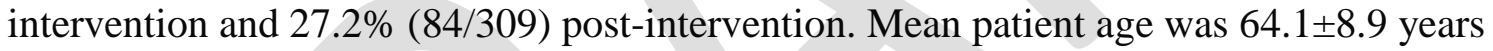
with no difference across time periods $(\mathrm{p}=0.39)$. Studies were performed for: $37.2 \%$ (115/309) elevated prostate serum antigen (PSA)/abnormal digital rectal exam (DRE) with previously negative template biopsy, 60.5\% (187/309) active surveillance (AS) and $1.6 \%$ (5/309) pre-biopsy. There was no difference in indications across time periods $(\mathrm{p}=0.84)$. Reporting templates were not used pre-intervention, increased to $38.3 \%$ (44/115) with intervention and 60.7\% (51/84) post-intervention, $(\mathrm{p}<0.001)$. Only 4.5\% (5/110) of reports included PI-RADS-version. 2 scores pre-intervention compared to 43.5\% (50/115) with intervention and 59.5\% (50/84) post-intervention, ( $\mathrm{p}<0.001)$. PIRADS-version.2 score use increased from $17.8 \%$ (38/214) without templates to $69.6 \%$ (66/95) with templates, $(\mathrm{p}<0.001)$. Radiologists were less likely to provide PI-RADSversion.2 scores for -MRI (42.1\% [90/214] +MRI versus 14.7\% [14/95] -MRI, $\mathrm{p}<0.001)$.

There was no difference in +MRI across time periods (67.3\% [74/110] +MRI preintervention, 68.7\% [79/115] +MRI intervention and 72.6\% [61/84] +MRI postintervention, $\mathrm{p}=0.52) .55 .6 \%(119 / 214)$ of men with + MRI underwent TB (58.1\% [43/74] pre-intervention, 55.7\% [44/79] in intervention and 52.5\% [32/61] postintervention). There was no difference in number of TB performed across time periods, $(p=0.47)$. Time interval between + MRI and TB decreased across time periods $(102 \pm 104$ days pre-intervention, $84 \pm 53$ days intervention and $62 \pm 33$ days post-intervention, 
$\mathrm{p}=0.028)$, Figure 2. 44.4\% (95/214) men with +MRI had no TB, including PI-RADSversion. 2 score 3 lesions and 77.9\% (74/95) of patients who were enrolled in AS.

\section{Discussion}

In this study, a simple intervention improved the use of prostate MRI reporting templates and PI-RADS-version.2 scoring. An association between reduced time from +MRI and subsequent TB, likely indicates enhanced communication between Urologists and Radiologists. The simple strategy of a standardized prostate MRI report which includes “pick-list” options for PI-RADS-version.2 scores could be implemented in most Radiology Departments.

The success of this study is multi-faceted. First, relates to ease of use of the new system, allowing Radiologists to use a predetermined “pick list” for PI-RADS-version.2 scores. Second, may be how the Radiologists in this study viewed structured reporting positively, overall (templates are commonly used at our institution) and after reenforcement through feedback from Urologists. Hawkins et $\mathrm{al}^{4}$ demonstrated Radiologists preferred structured reports after an internal review. Similarly, Stilseth et al $^{5}$ showed that Urologists prefer fully structured prostate MRI reporting, whereas, Radiologists prefer hybrid reporting. Radiologist preference may be a potential roadblock towards universal adoption of structured reporting; however, through education and feedback could be improved. Rosenkrantz et $\mathrm{al}^{6}$ showed the benefit of long-term follow-up after intervention to maintain adherence to providing a summary score on prostate MRI.

Radiologists in our study seldom provided PI-RADS-version. 2 scores for negative studies, exposing a potential lack of understanding regarding use of PI-RADS-version.2. Stilseth et $\mathrm{al}^{5}$ showed that among surveyed Radiologists and Urologists, roughly half thought PI-RADS-version.2 was not applied correctly by Radiologists. Impediments to use were speculated to be due to Urologist/Radiologist inexperience and lack of standardized template reporting. Larson et $\mathrm{al}^{7}$ found consensus-building efforts to be critical in development and implementation of structured reports, and that departmentwide structured reporting can be implemented in such a way that Radiologists prefer to use the standard reports.

PI-RADS-version.2 and the present study aim to enhance communication between Radiologists and Urologists through the use of probability scores. Our study indicates that PI-RADS-version. 2 scores improve communication, with a significant decrease in time between + MRI and TB without any other differences in patient access to account for the observation. In our study, roughly $40 \%$ of +MRI had no immediate TB, likely related to high number of AS patients in our cohort and inclusion of PI-RADS-version. 2 score 3 lesions, where biopsy could be delayed to coincide with AS protocols when there is stability in other clinical factors. Barentsz et $\mathrm{al}^{8}$ stress that PI-RADS-version.2 scores must be incorporated with clinical factors when determining need and strategy for biopsy. 


\section{Conclusion}

Our study demonstrates that through education, the use structured reporting templates, "pick-list” options for PI-RADS-version.2 assessment categories and through user feedback and support from urologists, the use of PI-RADS-version.2 can be improved in clinical practice.

\section{References}

1. American College of Radiology (ACR). MR prostate imaging and reporting and data system, version 2.0. www.acr.org. Published 2015. Date accessed Sept. 20, 2017.

2. Weinreb JC, Barentsz JO, Choyke PL, et al. PI-RADS prostate imaging reporting and data system: 2015, version 2. Eur Urol 2016; 69: 16-40.

3. Rosenkrantz AB, Ginocchio LA, Cornfeld D, et al. Interobserver Reproducibility of the PI-RADS Version 2 Lexicon: A Multicenter Study of Six Experienced Prostate Radiologists. Radiology 2016; 280(3): 793-804.

4. Hawkins CM, Hall S, Hardin J, Salisbury S, Towbin AJ. Prepopulated Radiology Report Templates: A Prospective Analysis of Error Rate and Turnaround Time. $J$ Digit Imaging 2012; 25(4): 504-11.

5. Stilseth B, Ghai S, Patel NU. A comparison of of Radiologists' and Urologists' Opinions Regarding Prostate MRI Reporting: Results From a Survey of Specialty Societies. AJR 2018; 210: 101-7.

6. Rosenkrantz AB, Pujara AC, Taneja SS. Use of a Quality Improvement Initiative to Achieve Consistent Reporting of Level of Suspicion for Tumor on Multiparametric Prostate MRI. AJR 2016; 206 (5): 1040-4

7. Larson DB, Towbin AJ, Pryor RM. Improving Consistency in Radiology Reporting through the Use of Department-wide Standardized Structured Reporting. Radiology 2013; 267(1): 240-50

8. Barentsz JO, Weinreb JC, Verma S, et al. Synopsis of the PI-RADS v2 Guidelines for Multiparametric Prostate Magnetic Resonance Imaging and Recommendations for Use. Eur Urol 2016: 69 (1): 41-9 


\section{Figures and Tables}

Fig. 1. Summary of patient inclusion and exclusion criteria. $\mathrm{P} 1=$ time period to intervention; $\mathrm{P} 2=$ time period during intervention; $\mathrm{P} 3=$ time period after intervention during feedback. MRI: magnetic resonance imaging.

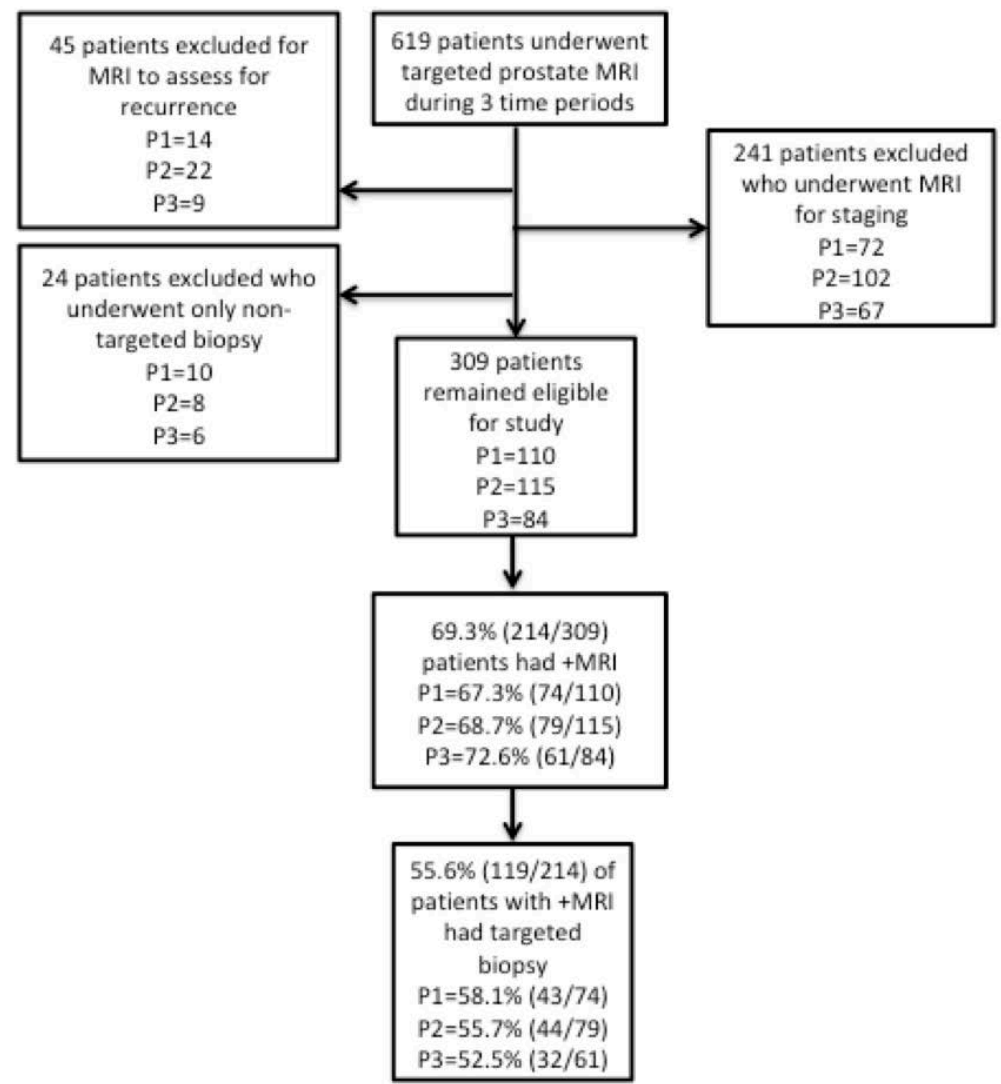


Fig. 2. Box and whisker plots showing the mean time between positive magnetic resonance imaging (MRI) examinations and subsequent biopsy decreased in this study through increased use of standardized reporting templates and PI-RADS v2 assessment categories with no differences in patient access in the three study periods to otherwise explain the finding.

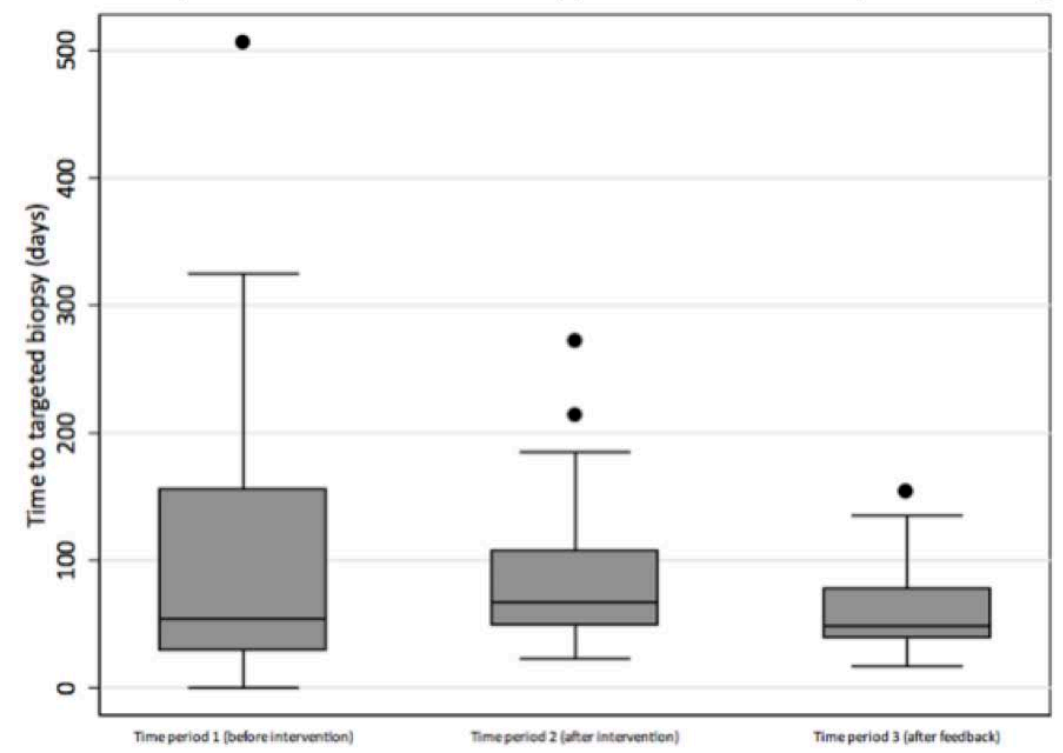

Table 1. Summary of Prostate Imaging and Data Reporting System (PI-RADS) version 2 assessment categories

\begin{tabular}{|l|r|}
\hline $\begin{array}{l}\text { Assessment } \\
\text { category }\end{array}$ & \\
\hline 1 & Very low (clinically significant cancer is highly unlikely to be present) \\
\hline 2 & Low (clinically significant cancer is unlikely to be present) \\
\hline 3 & Intermediate (the presence of clinically significant cancer is equivocal) \\
\hline 4 & High (clinically significant cancer is likely to be present) \\
\hline 5 & Very high (clinically significant cancer is highly likely to be present) \\
\hline
\end{tabular}


Supplementary Fig. 1. Illustration of standardized reporting template for magentic resonance imaging used at out institution in commercially available software (Powerscribe 360, Nuance Communications) to improve use of PI-RADS v2. A mandatory field under the "Impression" heading was created (red box) where radiologists are required to populate a PI-RADS v2 template field assigning the appropriate PI-RADS v2 assessment category using a "pick-list" tool (white arrow).

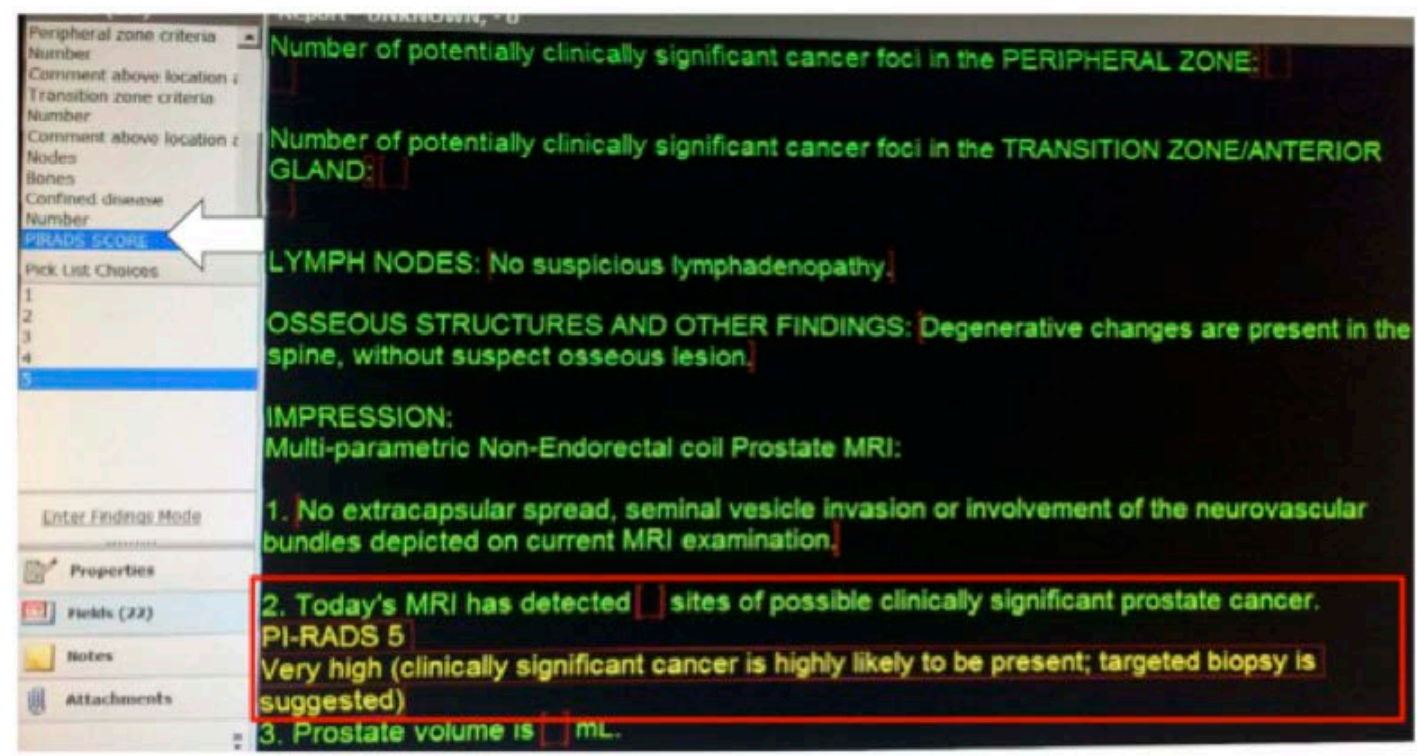

